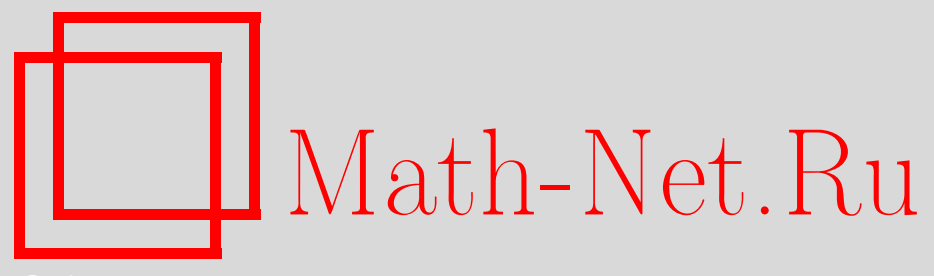

А. М. Селицкий, Пространство начальных данных 3-й краевой задачи для параболического дифференциальноразностного уравнения в одномерном случае, Матем. заметки, 2012, том 92, выпуск 4, 636-640

DOI: https://doi.org/10.4213/mzm9891

Использование Общероссийского математического портала Math-Net.Ru подразумевает, что вы прочитали и согласны с пользовательским соглашением http://www . mathnet.ru/rus/agreement

Параметры загрузки:

IP : 3.81 .55 .215

26 апреля 2023 г., $18: 17: 47$

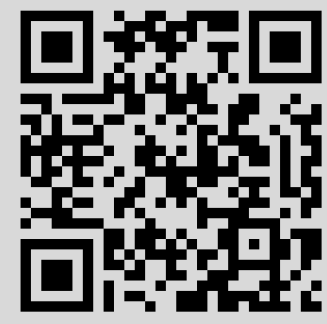




\section{Пространство начальных данных 3-й краевой задачи для параболического дифференциально-разностного уравнения в одномерном случае}

\section{А. М. Селицкий}

1. Постановка задачи. Пусть $Q=(0, d)$, положим $d=N+\theta$, где $N$ - целое положительное число, $0<\theta \leqslant 1$. При $\theta=1$ интервал $Q$ разобьем на интервалы

$$
Q_{1 k}=(k-1, k), \quad k=1, \ldots, N ;
$$

при $\theta<1$ - на интервалы

$$
Q_{1 k}=(k-1, k-1+\theta), \quad k=1, \ldots, N+1, \quad Q_{2 k}=(k-1+\theta, k), \quad k=1, \ldots, N .
$$

Введем ограниченные разностные операторы $R_{2}, R_{1}, R_{0}: L_{2}(\mathbb{R}) \rightarrow L_{2}(\mathbb{R})$ по формулам

$$
\left(R_{2} u\right)(x)=\sum_{k=-N}^{N} a_{k} u(x+k), \quad\left(R_{1} u\right)(x)=\sum_{k=-N}^{N} b_{k} u(x+k), \quad\left(R_{0} u\right)(x)=\sum_{k=-N}^{N} c_{k} u(x+k),
$$

где $a_{k}, b_{k}, c_{k}$ - комплексные числа.

Введем линейные ограниченные операторы $I_{Q}: L_{2}(Q) \rightarrow L_{2}(\mathbb{R})$ - оператор продолжения функции нулем вне $Q, P_{Q}: L_{2}(\mathbb{R}) \rightarrow L_{2}(Q)$ - оператор проекции функции на интервал $Q$, и операторы $R_{i Q}: L_{2}(Q) \rightarrow L_{2}(Q)$, определенные равенствами

$$
R_{i Q}=P_{Q} R_{i} I_{Q}, \quad i=0,1,2 .
$$

Будем рассматривать следующую задачу:

$$
\begin{gathered}
u_{t}-\left(R_{2 Q} u_{x}\right)_{x}+R_{1 Q} u_{x}+R_{0 Q} u=f(x, t), \quad(x, t) \in Q_{T}=Q \times(0, T), \\
\left.\left(-R_{2 Q} u_{x}+\alpha u\right)\right|_{x=0}=0,\left.\quad\left(R_{2 Q} u_{x}+\beta u\right)\right|_{x=d}=0, \quad 0<t<T, \\
\left.u\right|_{t=0}=\varphi(x), \quad x \in Q,
\end{gathered}
$$

где $f \in L_{2}\left(Q_{T}\right), \varphi \in L_{2}(Q), \alpha \geqslant 0, \beta \geqslant 0,0<T<\infty$.

Чтобы сформулировать определение сильного решения задачи (1)-(3), введем неограниченный оператор $A: L_{2}(Q) \rightarrow L_{2}(Q)$, действующий по формуле

$$
A v=-\left(R_{2 Q} v_{x}\right)_{x}+R_{1 Q} v_{x}+R_{0 Q} v, \quad v \in D(A),
$$

с областью определения, состоящей из функций $v \in W_{2}^{1}(Q)$ (через $W_{2}^{\ell}(Q)$ будем обозначать пространство Соболева комплекснозначных функций) таких, что $v \in W_{2}^{2}\left(Q_{s k}\right)$, где $Q_{s k}-$ интервалы, на которые мы разбили $Q$, и удовлетворяющих условиям

$$
\begin{gathered}
\left.R_{2 Q} v_{x}\right|_{x=k+0}=\left.R_{2 Q} v_{x}\right|_{x=k-0}, \\
\left.R_{2 Q} v_{x}\right|_{x=\theta+k-1+0}=\left.R_{2 Q} v_{x}\right|_{x=\theta+k-1-0} \quad(\text { при } \quad \theta<1), \quad k=1, \ldots, N, \\
\left.\left(-R_{2 Q} v_{x}+\alpha v\right)\right|_{x=0}=0,\left.\quad\left(R_{2 Q} v_{x}+\beta v\right)\right|_{x=d}=0,
\end{gathered}
$$

T.e.

$$
D(A)=\left\{v \in W_{2}^{1}(Q): v \in W_{2}^{2}\left(Q_{s k}\right) \text { и выполняются (5) и (6) }\right\} .
$$

Работа выполнена при поддержке Российского фонда фундаментальных исследований (грант № 10-01-00837).

(C) А. М. СЕлицкий, 2012 
ОПредЕлениЕ 1. Оператор $A$ будем называть сильно эллиптическим, если существуют числа $c_{1}>0$ и $c_{2} \geqslant 0$ такие, что

$$
\operatorname{Re}(A v, v)_{L_{2}(Q)} \geqslant c_{1}\|v\|_{W_{2}^{1}(Q)}^{2}-c_{2}\|v\|_{L_{2}(Q)}^{2}, \quad v \in D(A) .
$$

Лемма 1.2 в [1] дает необходимое и достаточное условие сильной эллиптичности оператора $A$.

Лемма 1. Для того, чтобы оператор А был силъно эллиптическим, необходимо и достаточно, чтобы матрича $R+R^{*}$ была положительно определена, где $R=\left\|a_{j-i}\right\|_{i, j=1}^{N+1}$, a $R^{*}$ - эрмитово сопряженная матрица.

В дальнейшем будем предполагать, что оператор $A$ сильно эллиптический. Заметим также, что вместо оператора $A$ можно рассматривать оператор $A+c_{2} I$, что соответствует замене функции $u$ в задаче (1)-(3) на $\widetilde{u}=e^{c_{2} t} u$, а тогда в неравенстве (7) можно считать $c_{2}=0$.

Несложно проверить, что при предположении, что выполняется неравенство (7) с $c_{2}=0$, оператор $(-A)$ (а следовательно, и оператор $A$ ) является замкнутым (см., например, теорему 4.5 гл. 1 в [2]), а тогда пространство $D(A)$ является гильбертовым с нормой графика. Введем гильбертово пространство

$$
W(A)=\left\{w \in L_{2}([0, T] ; D(A)): w_{t} \in L_{2}\left(Q_{T}\right)\right\} .
$$

ОПредЕление 2. Силъным решением задачи (1)-(3) будем называть функцию $u \in$ $W(A)$, удовлетворяющую уравнению $(1)$ при почти всех $(x, t)$ в $Q_{T}$ и начальному условию (3).

Из теоремы 2.2 в [1] следует, что задача (1)-(3) эквивалентна абстрактному операторному уравнению

$$
\frac{d u}{d t}+A u=f(t), \quad u(0)=\varphi,
$$

рассматриваемому в $L_{2}\left([0, T] ; L_{2}(Q)\right)=L_{2}\left(Q_{T}\right)$.

Пусть $X$ и $Y$ - банаховы пространства и $X \subset Y$ плотно и непрерывно. Обозначим через $S$ полосу $S=\{z: 0<\operatorname{Re} z<1\}$. Через $\mathscr{H}(X, Y)$ обозначим пространство непрерывных ограниченных функций $F: \bar{S} \rightarrow Y$ аналитических в $S$ таких, что $F(i y) \in X$ непрерывна и ограничена, как $X$-значная функция от $y$, а $F(1+i y) \in Y$ непрерывна и ограничена, как $Y$-значная функция от $y$, с нормой

$$
\|F\|_{\mathscr{H}(X, Y)}=\max \left\{\sup _{-\infty<y<+\infty}\|F(i y)\|_{X}, \sup _{-\infty<y<+\infty}\|F(1+i y)\|_{Y}\right\} .
$$

ОПРеДЕЛЕНИЕ 3. Интерполяиионным пространством $[X, Y]_{\theta}$ называется образ пространства $\mathscr{H}(X, Y)$ при отображении $F \rightarrow F(\theta)$ :

$$
[X, Y]_{\theta}=\{f \in Y: \exists F(z) \in \mathscr{H}(X, Y) \text { такая, что } F(\theta)=f\},
$$

с нормой

$$
\|f\|_{[X, Y]_{\theta}}=\inf _{F(\theta)=f}\|F\|_{\mathscr{H}(X, Y)} .
$$

Подробнее о комплексном методе интерполяции см., гл. 1 в [3] или [4].

Tеорема 1. Пустъ оператор А сильно эллиптический с $c_{2}=0$. Тогда для любых $f \in L_{2}\left(Q_{T}\right)$ и $\varphi \in\left[D(A), L_{2}(Q)\right]_{1 / 2}$ задача (1)-(3) имеет единственное сильное решение, которое определяется по формуле

$$
u(x, t)=T_{t} \varphi(x)+\int_{0}^{t} T_{t-s} f(x, s) d s,
$$

где $\left\{T_{t}\right\}, t \geqslant 0$ - аналитическая полугруппа с генератором $(-A)$.

ДокАЗАТЕЛЬСтво следует из теорем 3.1 и 3.2 в [1]. 
2. Пространство начальных данных. В теореме 1 условие принадлежности начальной функции пространству $\left[D(A), L_{2}(Q)\right]_{1 / 2}$ является необходимым и достаточным условием существования сильного решения (см. [5]).

Обозначим через $W_{2}^{\ell, R}(Q)$ (соответственно $W_{2}^{\ell, R}(Q)$ ) множество функций $v \in W_{2}^{\ell}(Q)$ таких, что $v \in \stackrel{\circ}{W}_{2}^{\ell}\left(Q_{s k}\right)$ (соответственно $\left.v \in W_{2}^{\ell}\left(Q_{s k}\right)\right)$. Тогда

$$
\stackrel{\circ}{W}_{2}^{1, R}(Q)=\left[\stackrel{\circ}{W}_{2}^{2, R}(Q), L_{2}(Q)\right]_{1 / 2}, \quad W_{2}^{1, R}(Q)=\left[W_{2}^{2, R}(Q), L_{2}(Q)\right]_{1 / 2} .
$$

Tеорема 2. Пустъ оператор А сильно эллиптический с $c_{2}=0$. Тогда

$$
\left[D(A), L_{2}(Q)\right]_{1 / 2}=W_{2}^{1}(Q) .
$$

ДокАзАтЕльство. Доказательство вложения $\left[D(A), L_{2}(Q)\right]_{1 / 2} \subset W_{2}^{1}(Q)$ аналогично доказательству теоремы 4.3 в [6]. Докажем обратное вложение. Пусть $f \in W_{2}^{1}(Q)$. Покажем, что существует функция $F \in \mathscr{H}\left(D(A), L_{2}(Q)\right)$ такая, что $F(1 / 2)=f$. Пусть функция $v \in D(A)$ и $\left.v\right|_{\partial Q_{s k}}=\left.f\right|_{\partial Q_{s k}}$. Положим $F_{1} \equiv v$. Тогда $F_{1}(1 / 2)-f \in \mathscr{W}_{2}^{1, R}(Q)$ и существует функция $F_{2} \in \stackrel{\circ}{H}\left(\stackrel{\circ}{W}_{2}^{2, R}(Q), L_{2}(Q)\right)$ такая, что $F_{2}(1 / 2)=F_{1}(1 / 2)-f$. Положим $F=F_{1}+F_{2}$, имеем $F \in \mathscr{H}\left(D(A), L_{2}(Q)\right)$, так как $\stackrel{\circ}{2}_{2}^{2, R}(Q) \subset D(A)$.

Для завершения доказательства построим явно функцию $v \in D(A)$ такую, что $\left.v\right|_{\partial Q_{s k}}=$ $\left.f\right|_{\partial Q_{s k}}$. Рассмотрим случай $\theta<1$. Обозначим $L_{2}\left(\bigcup_{k} Q_{s k}\right)$ - подпространство функций из $L_{2}(Q)$, равных нулю вне

$$
\bigcup_{k} Q_{s k}, \quad s=1,2, \quad k=1, \ldots, N_{s}, \quad N_{1}=N+1, \quad N_{2}=N .
$$

Оператор ортогонального проектирования на $L_{2}\left(\bigcup_{k} Q_{s k}\right)$ обозначим через $P_{s}$. Введем оператор, осуществляющий изоморфизм гильбертовых пространств

$$
U_{s}: L_{2}\left(\bigcup_{k} Q_{s k}\right) \rightarrow \prod_{k} L_{2}\left(Q_{s 1}\right)
$$

по формуле

$$
\left(U_{s} u\right)_{k}(x)=u(x+k-1), \quad x \in Q_{s 1} .
$$

В силу леммы 2.6 в [7; $§ 2]$, операторы $R_{2 Q s}=U_{s} R_{2 Q} U_{s}^{-1}$ являются операторами умножения на матрицы. Рассмотрим векторы

$$
\begin{array}{ll}
V_{1}=\left.\left(U_{1} P_{1} v_{x}\right)\right|_{x=0+}, & V_{2}=\left.\left(U_{2} P_{2} v_{x}\right)\right|_{x=1-0}, \\
V_{3}=\left.\left(U_{1} P_{1} v_{x}\right)\right|_{x=\theta-0}, & V_{4}=\left.\left(U_{2} P_{2} v_{x}\right)\right|_{x=\theta+0} .
\end{array}
$$

Тогда условия (5), (6) можно записать в виде

$$
\begin{aligned}
& \left(\begin{array}{ccc}
a_{0} & \ldots & a_{N} \\
a_{-1} & \ldots & a_{N-1} \\
\ldots \ldots & \ldots & \ldots \\
a_{-N+1} & \ldots & a_{1}
\end{array}\right)\left(\begin{array}{c}
V_{31} \\
V_{32} \\
\ldots \\
V_{3, N+1}
\end{array}\right)-\left(\begin{array}{ccc}
a_{0} & \ldots & a_{N-1} \\
a_{-1} & \ldots & a_{N-2} \\
\ldots \ldots & \ldots \ldots & \ldots \ldots \\
a_{-N+1} & \ldots & a_{0}
\end{array}\right)\left(\begin{array}{c}
V_{41} \\
V_{42} \\
\ldots \\
V_{4 N}
\end{array}\right)=0, \\
& \left(a_{-N} a_{-N+1} \ldots a_{0}\right) V_{3}+\left.\beta v\right|_{x=d-0}=0 . \\
& \left(\begin{array}{ccc}
a_{-1} & \ldots & a_{N-1} \\
a_{-2} & \ldots & a_{N-2} \\
\ldots \ldots & \ldots & \ldots \\
a_{-N} & \ldots & a_{0}
\end{array}\right)\left(\begin{array}{c}
V_{11} \\
V_{12} \\
\ldots \\
V_{1, N+1}
\end{array}\right)-\left(\begin{array}{ccc}
a_{0} & \ldots & a_{N-1} \\
a_{-1} & \ldots & a_{N-2} \\
\ldots \ldots \ldots \ldots \ldots & \ldots \ldots \\
a_{-N+1} & \ldots & a_{0}
\end{array}\right)\left(\begin{array}{c}
V_{21} \\
V_{22} \\
\ldots \\
V_{2 N}
\end{array}\right)=0, \\
& -\left(a_{0} a_{1} \ldots a_{N}\right) V_{1}+\left.\alpha v\right|_{x=0+}=0 .
\end{aligned}
$$


В силу условия сильной эллиптичности оператора $A$ матрица $R$ положительно определена, а тогда, положив $\left.v\right|_{x=0+}=\left.f\right|_{x=0+},\left.v\right|_{x=d-0}=\left.f\right|_{x=d-0}$, получаем, что системы $(10),(11)$ и (12), (13) имеют (вообще говоря, не единственные) решения $V_{1}, V_{2}, V_{3}, V_{4}$.

Рассмотрим функцию $w \in L_{2}(Q)$, определенную следующим равенством:

$$
w(x)= \begin{cases}V_{1 k}(x-k+1)+\left.f\right|_{x=k-1+0}, & x \in\left(k-1, k-1+\frac{\theta}{2}\right), \\ V_{3 k}(x-k+1-\theta)+\left.f\right|_{x=k-1+\theta-0}, & x \in\left(k-1+\frac{\theta}{2}, k-1+\theta\right), \\ V_{4 k}(x-k+1-\theta)+\left.f\right|_{x=k-1+\theta+0}, & x \in\left(k-1+\theta, k-\frac{\theta}{2}\right), \\ V_{2 k}(x-k)+\left.f\right|_{x=k-0}, & x \in\left(k-\frac{\theta}{2}, k\right),\end{cases}
$$

т.е. функция $w$ состоит из отрезков прямых, имеющих на границах отрезка $Q_{s k}$ коэффициент наклона $V_{i k}, i=1,2,3$ или 4 , и принимающие значения, которые принимает функция $f$.

На отрезке $Q$ зададим функции $\xi_{1 k} \in \dot{C}^{\infty}(\bar{Q})$ такие, что

$$
\begin{array}{lll}
\xi_{1 k}(x)=1 & \text { при } & x \in[k-1+2 \delta, k-1+\theta-2 \delta], \\
\xi_{1 k}(x)=0 & \text { при } & x \notin(k-1+\delta, k-1+\theta-\delta), \quad \delta=\frac{\theta}{4},
\end{array}
$$

а также функции $\xi_{2 k} \in \dot{C}^{\infty}(\bar{Q})$ такие, что

$$
\begin{array}{ll}
\xi_{2 k}(x)=1 & \text { при значениях } \quad x \in[k-1+\theta+2 \sigma, k-2 \sigma], \\
\xi_{2 k}(x)=0 & \text { при } \quad x \notin(k-1+\theta+\sigma, k-\sigma), \quad \sigma=\frac{1-\theta}{4} .
\end{array}
$$

Положим

$$
v(x)=w(x) \cdot \sum_{s, k}\left(1-\xi_{s k}(x)\right) .
$$

По построению $v \in D(A)$ и $\left.v\right|_{\partial Q_{s k}}=\left.f\right|_{\partial Q_{s k}}$.

ЗАмечаниЕ. В гл. VI монографии [8] Като была сформулирована проблема: "Верно ли, что $D\left(A^{1 / 2}\right)$ и $D\left(\left(A^{*}\right)^{1 / 2}\right)$ совпадают?" В [9] был построен пример аккретивного оператоpa, не удовлетворяющего данному условию, в [10] был построен пример $m$-секториального оператора $\mathscr{A}$, для которого $D\left(\mathscr{A}^{1 / 2}\right) \neq D\left(\mathscr{A}^{* 1 / 2}\right)$. Положительное решение этого вопроса для сильно эллиптических систем дифференциальных уравнений с измеримыми коэффициентами в $\mathbb{R}^{n}$ было дано в [11], а для сильно эллиптических уравнений в липшицевых областях - в [12]. В статье [13] гипотеза Като была доказана для широкого класса сильно эллиптических функционально-дифференциальных уравнений. При выполнении условий теоремы 2 справедливо равенство $D\left(A^{1 / 2}\right)=D\left(\left(A^{*}\right)^{1 / 2}\right)$ для операторов $A$, рассмотренных в настоящей работе (оператор $A^{*}$ определяется по полуторалинейной форме, связанной с оператором $A$, см., например, [14], и для $A^{*}$ справедлива теорема 3.1 настоящей работы).

Автор выражает благодарность А. Л. Скубачевскому за постановку задачи и постоянное внимание к работе, а также М. С. Аграновичу за указание на работы Р. Сили по комплексной интерполяции.

\section{СПИСОК ЦИТИРОВАННОЙ ЛИТЕРАТУРЫ}

[1] A. M. Selitskii, Funct. Differ. Equ., 14:2-4 (2007), 373-395. [2] A. Pazy, Semigroups of Linear Operators and Applications to Partial Differential Equations, Appl. Math. Sci., 44, Springer-Verlag, Berlin, 1983. [3] Ж.-Л. Лионс, Э. Мадженес, Неоднородные граничные задачи и их приложения, Т. 1, Мир, М., 1971. [4] R. Seeley, Studia Math., 44 (1972), 47-60. 
[5] A. Ashyralyev, P. E. Sobolevskii, Well-Posedness of Parabolic Difference Equations, Oper. Theory Adv. Appl., 69, Birkhäuser-Verlag, Basel, 1994. [6] A. L. Skubachevskii, R. V. Shamin, Funct. Differ. Equ., 8:3-4 (2001), 407-424. [7] A. L. Skubachevskii, Elliptic Functional Differential Equations and Applications, Oper. Theory Adv. Appl., 91, Birkhäuser-Verlag, Basel, 1997. [8] Т. Като, Теория возмущений линейных операторов, Мир, M., 1972. [9] J.L. Lions, J. Math. Soc. Japan, 14:2 (1962), 233-241. [10] A. McIntosh, Proc. Amer. Math. Soc., 32 (1972), 430-434. [11] P. Auscher, S. Hofmann, A. McIntosch, P. Tchamitchian, J. Evol. Equ., 1:4 (2001), 361-385. [12] A. Axelsson, S. Keith, A. McIntosh, J. London Math. Soc. (2), 74:1 (2006), 113-130. [13] Р. В. Шамин, Матем. сб., 194:9 (2003), 141-156. [14] Е. Л. Цветков, Матем. заметки, 51:6 (1992), 107-114.

\section{А. М. Селицкий}

Поступило

Вычислительный центр

20.04.2012

им. А. А. Дородницына РАН, г. Москва

E-mail: selitsky@mail.ru 